\title{
HOW NORMAL ARE THE PECULIAR A STARS
}

\section{G.S.D.BABU}

Indian Institute of Astrophysics, Bangalore 560034, India

\section{B.S.SHYLAJA}

Physical Research Laboratory, Ahmedabad 380009, India

\begin{abstract}
A comparison of various physical parameters of the normal $A$ stars with those obtained by using the peculiarityfree zones of $\mathrm{Ap}$ stars indicate that the peculiar A stars may be treated as normal stars as far as their effective temperatures, radii, bolometric corrections and the mass range are concerned.
\end{abstract}

\section{INTRODUCTION}

The very fact that these stars are essentially classified as belonging to type A, implies that their basic parameters are expected to be very much like those of normal A stars. However, the presence of some peculiar features, which are over and above the normal characteristics, place them in the special category of Ap type. Many authors have already shown the variety of differences between the peculiar and normal A stars. For example, Gerbaldi (1974) pointed out that the Balmer discontinuity in Ap stars is affected by the particular character of the star, while Megessier (1988) found that the Ap stars have less UV flux and a larger visible flux along with a smaller Balmer jump as compared to the normal stars of the same effective temperature. Faraggiana (1987) emphasised that the UBV indices of Ap stars are similar to those of normal stars only by chance, and have no direct relation to $T_{e}$, and Radkov (19SS) found their energy distributions to be near the normal class IV stars. Then Kroll (1987) and Kroll et al. (1987) indicated that the Ap stars behave like main sequence objects and hence like black bodies in the near IR, where they are not affected by the chemical peculiarities. Further, the now well known 4200 and 5200 features, which are seen in many Ap stars, have 
attracted the attention of several authors. Thus, in order to assess the normality of these peculiar stars, only the peculiarity-free zones like the longer wavelength regions are to be utilised and based on the extent of normality, the specificities of these stars may be confidently accounted for. This is important for its consequence, mainly concerning the evolutionary stage of these stars. With this in view, an attempt has been made to find out how normal the peculiar A stars are. For the purpose of this work, the sample of stars listed in our earlier paper (Babu \& Shylaja, 1981) has been chosen, where $\mathrm{T}(\mathrm{eff}), \mathrm{R} / \mathrm{R}$ (sun) and bolometric corrections of more than $100 \mathrm{Ap}$ stars are given. It may be noted here that all these values have been determined using homogeneous data; the differences in the values obtained by employing the earlier models and the ones given by Kurucz (1979) are found to be non-critical in this work.

\section{RELATIONSHIP BETWEEN M(vis) AND R/R(sun)}

Since it is known that the radius of a given star is directly related to its absolute magnitude, the $\mathrm{M}$ (vis) of Ap stars is plotted in Fig.1 against their respective $\log R / R$ (sun), along with a few normal $A$ stars. In this figure, the normal and the peculiar A stars appear to be following the same trend as that of the main sequence stars. However, the brighter stars seem to be showing a tendency towards the larger size.

\section{RELATIONSHIP BETWEEN T(eff) AND BOLOMETRIC CORRECTION}

It has been pointed out by earlier authors that the bolometric correction is a function of temperature for stars hotter than FO type. This is clearly seen in Fig.2, which is a plot between Theta(eff $)=5040 / T($ eff $)$ and the bolometric corrections of the respective Ap stars. Even the normal A stars are following the same relationship. It is interesting to note that the bolometric corrections given by Kurucz (1979) for $\log g=4.0$ and $\log$ $A=-1.0$ also appear to be matching very well with the observations. 


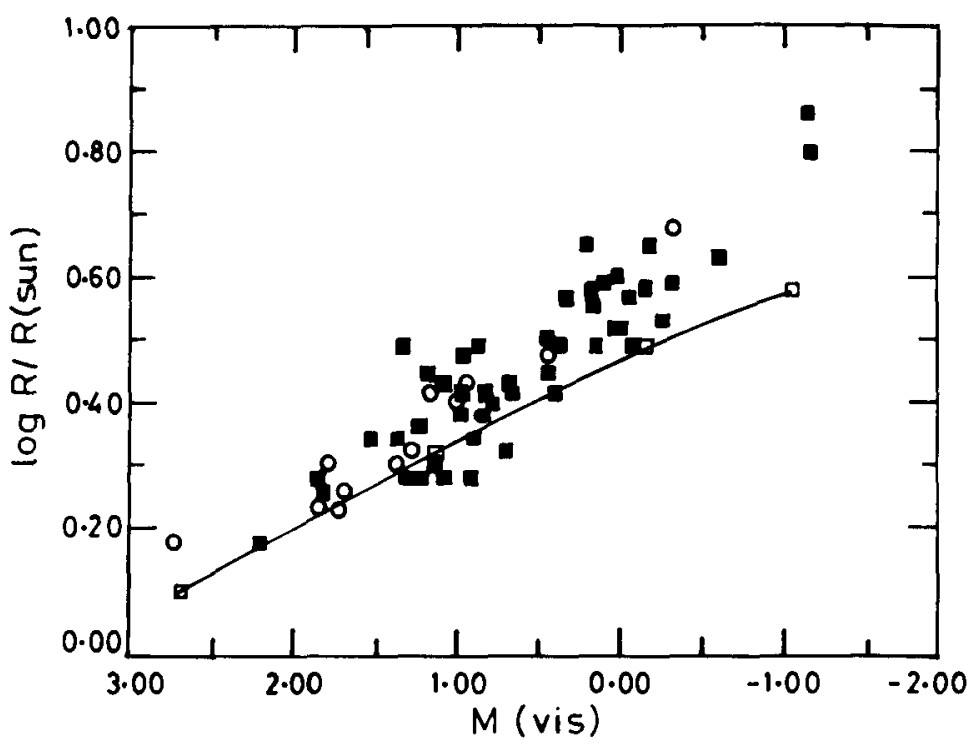

Fig.1. Relationship between absolute magnitudes and radii of $A p$ and normal A stars. The filled squares denote the Ap stars, while the circles represent the normal A stars. The solid line is the locus of the main sequence stars as given by Allen (1973).

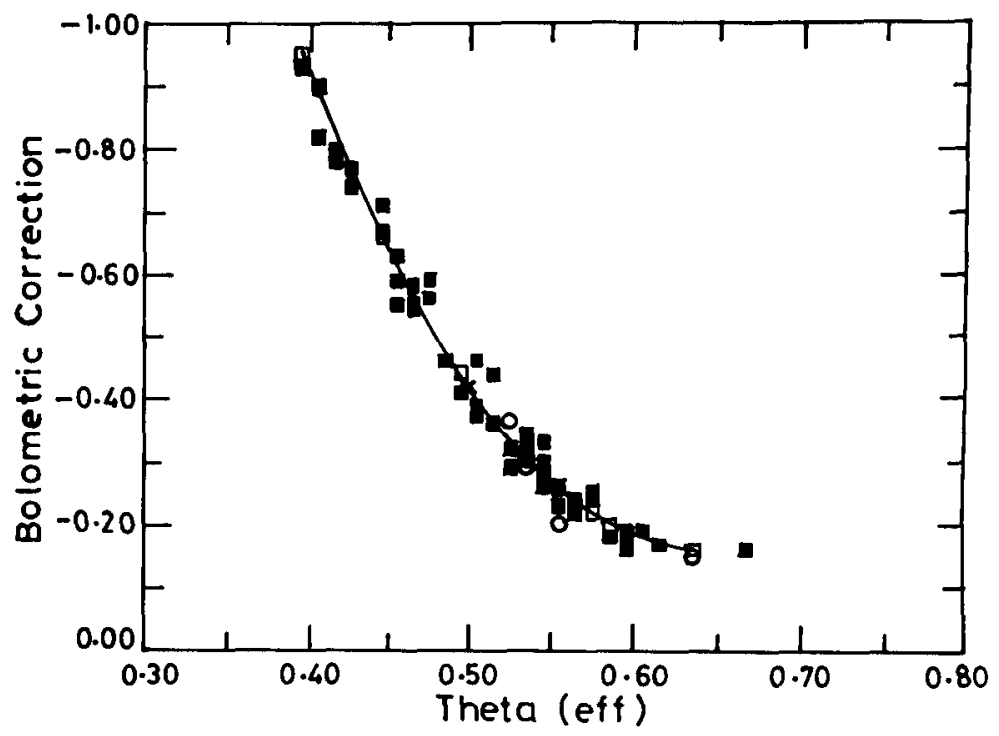

Fig.2. Relationship between the effective temperatures and the bolometric corrections of Ap and normal A stars. The symbols are same as in Fig.1, the solid line being the least squares solution of the observed relationship. 


\section{EVOLUTIONARY ASPECTS}

In order to study the evolutionary aspects of the Ap stars, a plot between their $\log T($ eff $)$ and the corresponding $M($ bol) is drawn in Fig.3. The location of the Ap stars in this diagram, which is essentially the HRdiagram, is found to be around the upper edge of the main sequence band with some stars situated even above the main sequence. Following the evolutionary tracks (taken from Iben, 1967), shown in the diagram, one is tempted to say that these stars are probably transiting towards the yellow giant region and are presently in the hydrogen shell burning phase. The isochrones also indicate that these stars are in the mass range of about 1.5 M(sun) to about 3.0 M(sun), which again is the same as that of the normal A stars at this evolutionary phase.

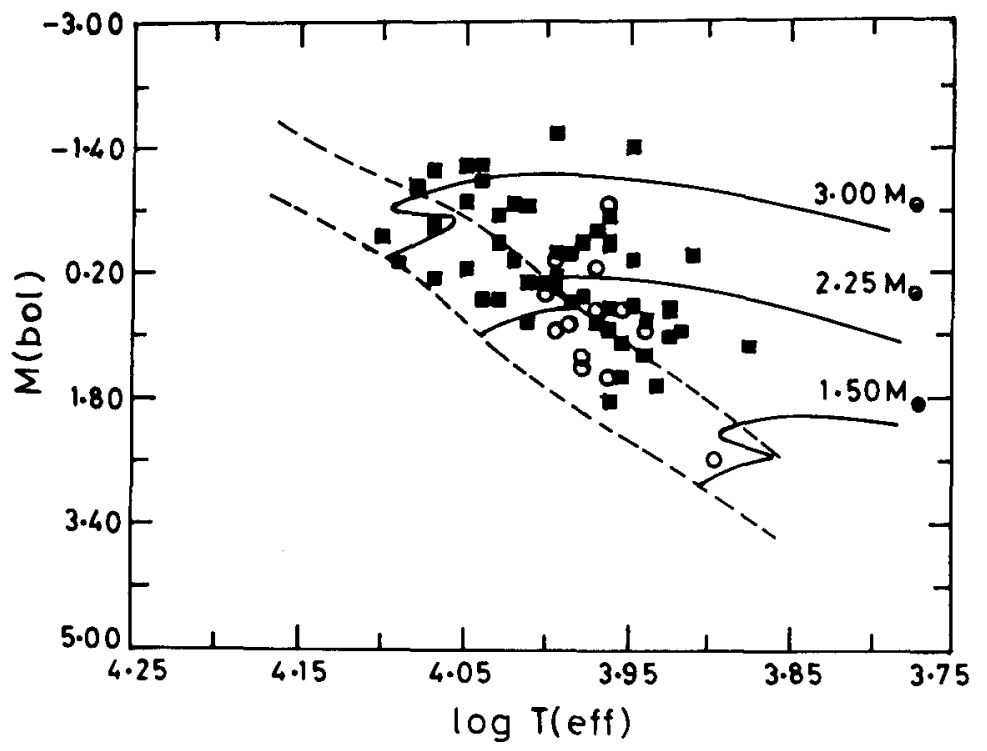

Fig.3. Relationship between effective temperatures and bolometric magnitudes of Ap and normal A stars. The symbols are same as in Fig.1. The dashed lines are the boundaries of the main sequence band and the solid lines are the evolutionary tracks. 
Thus, a clear indication emerges to show that the peculiar A stars may be treated as normal stars as far as their physical parameters like the effective temperature, radius, bolometric corrections and mass range are concerned.

\section{ACKNOWLEDGEMENTS}

I wish to express my gratitude to Prof.M.Hack and Prof.R.Faraggiana for all their support and encouragement in preparing this paper and for enabling me to attend this conference. I am thankful to the Organizing Committee of IAU Coll. 138 for granting me a major part of the financial assistance and to the Indian Institute of Astrophysics for their partial support.

\section{REFERENCES}

Allen,G.W. 1973, Astrophysical Quantities, Athlone Press, London.

Babu,G.S.D., Shylaja,B.S. 1981, Astrophys. Sp. Science, 79, 243.

Faraggiana,R. 1987, Astrophys. Sp. Science, 134, 381.

Gerbaldi,M. 1974, Astron. Astrophys., 30, 105.

Iben,I. Jr. 1967, Astrophys. J., 147, 624.

Kroll,R. 1987, Astron. Astrophys., 181, 315.

Kroll,R., Schneider,H., Catalano,F.A., Voigt,H.H. 1987, Astron. Astrophys. Suppl., 67, 195.

Kurucz,R.L. 1979, Astrophys. J. Suppl., 4, 1.

Loden,L.O., Sundman,A. 1987, J. Astrophys. Astron., 8, 351.

Megessier,C. 1988, Astron. Astrophys., 206, 74.

Radkov,R.S. 1988, Dokl. Bolg. Akad. Nauk., 41, 5. 